Check for updates

Cite this: Phys. Chem. Chem. Phys., 2020, 22, 10536

Received 29th November 2019 Accepted 24th January 2020

DOI: $10.1039 / c 9 c p 06474 e$

rsc.li/pccp

\section{Revisiting the Volmer-Heyrovský mechanism of hydrogen evolution on a nitrogen doped carbon nanotube: constrained molecular dynamics versus the nudged elastic band method $\dagger$}

\author{
Rasmus Kronberg, (D) Heikki Lappalainen and Kari Laasonen (D)* \\ Density functional theory (DFT) based computational electrochemistry has the potential to serve as a \\ tool with predictive power in the rational development and screening of electrocatalysts for renewable \\ energy technologies. It is, however, of paramount importance that simulations are conducted rigorously \\ at a level of theory that is sufficiently accurate in order to obtain physicochemically sensible results. \\ Herein, we present a comparative study of the performance of the static climbing image nudged elastic \\ band method (Cl-NEB) vs. DFT based constrained molecular dynamics simulations with thermodynamic \\ integration in estimating activation and reaction (free) energies of the Volmer-Heyrovský mechanism on \\ a nitrogen doped carbon nanotube. Due to cancellation of errors within the $\mathrm{Cl}$-NEB calculations, static \\ and dynamic activation barriers are observed to be surprisingly similar, while a substantial decrease \\ in reaction energies is seen upon incorporation of solvent dynamics. This finding is attributed to two \\ competing effects; (1) solvent reorganization that stabilizes the transition and, in particular, the product \\ states with respect to the reactant state and (2) destabilizing entropic contributions due to solvent \\ fluctuations. Our results highlight the importance of explicitly sampling the interfacial solvent dynamics \\ when studying hydrogen evolution at solid-liquid interfaces.
}

\section{Introduction}

Accurate simulations of electrochemical interfaces and reactions are becoming increasingly valuable as a complementary method in the rational design of sustainable approaches to energy conversion and storage. ${ }^{1-3}$ Especially the development of novel electrocatalysts for reactions such as hydrogen evolution/oxidation (HER/ HOR) and oxygen evolution/reduction (OER/ORR), important within proton exchange membrane electrolysers and fuel cells, can be augmented by density functional theory (DFT) based methods with the ability to elucidate atomistic details that experiments alone struggle to resolve. The field of computational electrochemistry is, however, still in its infancy and consequently applied methods and levels of theory require careful benchmarking for precise physicochemical predictions. ${ }^{4}$

Computational kinetic modelling of catalytic processes entails the determination of the transition path connecting the initial (reactant, IS) and final (product, FS) states. The most interesting quantities associated with the reaction path are the

Research Group of Computational Chemistry, Department of Chemistry and Materials Science, Aalto University, P.O. Box 16100, FI-00076 Aalto, Finland. E-mail: kari.laasonen@aalto.fi

$\dagger$ Electronic supplementary information (ESI) available. See DOI: 10.1039/c9cp06474e activation barrier $\left(E^{\ddagger}\right)$ and the reaction energy $(\Delta E)$, defined by the energy differences between the reactant and transition states (TS) and the reactant and product states, respectively. While the reaction energy quantifies the driving force of the concerned reaction, the rate determining step (RDS) and consequently the reaction kinetics are ultimately dictated by the activation barrier height. Within the framework of DFT simulations, transition path optimizations are frequently conducted using the nudged elastic band (NEB) method, ${ }^{5}$ especially in conjunction with the climbing image modification (CI-NEB) ${ }^{6}$ that drives the image with the highest energy up to the saddle point. While the CI-NEB method is successful in describing the transition paths of processes taking place e.g. at vacuum interfaces, ${ }^{7-9}$ complications arise, however, for reactions lacking well defined initial and final states. Particularly, finite-temperature electrochemical reactions occurring at solid-liquid interfaces are challenging to simulate using static transition search methods such as CI-NEB as the dynamic solvent degrees of freedom may play a decisive part in the reaction coordinate. ${ }^{10}$ Indeed, ignoring such fluctuations is highly questionable as they constitute a potentially important entropic contribution to the activation barrier.

Consequently, to simulate rare events in dynamic environments appropriate sampling of the fluctuating medium is needed. A rigorous approach for this purpose is the constrained 
molecular dynamics (MD) method introduced by Sprik and Ciccotti. ${ }^{11}$ This methodology enables the study of activated processes by enforcing a holonomic constraint on a reaction coordinate along which the reaction is assumed to progress. By performing thermodynamic integration on the average force of constraint unbiased by a geometric correction term, the free energy profile of the reaction can be elegantly computed. The constrained MD method has been consequently applied very successfully to study reactions such as proton transfer, ${ }^{12-16}$ hydrolysis ${ }^{17,18}$ and redox reactions of metal ions in solution, ${ }^{19,20}$ as well as simple interfacial processes. ${ }^{21-26}$ Nevertheless, to our knowledge DFT based constrained MD simulations have not been applied for the direct simulation of HER/HOR or OER/ORR as of yet. Instead, the kinetics of these within electrochemical energy conversion highly relevant reactions have been investigated atomistically employing only static approaches. ${ }^{27-35}$

This work presents a comparative study of the CI-NEB and constrained MD methodologies and their ability to describe the transition path and the associated potential energy surface of the Volmer-Heyrovský mechanism of HER. In acidic media, the Volmer-Heyrovský mechanism proceeds via two protoncoupled electron transfer (PCET) steps; (1) electrosorption of a solvated proton, i.e. the Volmer reaction, followed by (2) an Eley-Rideal-type recombination of the adsorbed hydrogen $\left(\mathrm{H}^{*}\right)$ and another solvated proton to form molecular hydrogen, the Heyrovský reaction.

$$
\begin{gathered}
{\left[\left(\mathrm{H}_{2} \mathrm{O}\right)_{n}-\mathrm{H}\right]^{+}+\mathrm{e}^{-} \rightleftharpoons \mathrm{H}^{*}+\left(\mathrm{H}_{2} \mathrm{O}\right)_{n}} \\
{\left[\left(\mathrm{H}_{2} \mathrm{O}\right)_{n}-\mathrm{H}\right]^{+}+\mathrm{H}^{*}+\mathrm{e}^{-} \rightleftharpoons \mathrm{H}_{2}+\left(\mathrm{H}_{2} \mathrm{O}\right)_{n}}
\end{gathered}
$$

In the preceding, the solvated proton complex is denoted by $\left[\left(\mathrm{H}_{2} \mathrm{O}\right)_{n}-\mathrm{H}\right]^{+}$, where $n=1,2$ and 4 are recognized as the hydronium $\left(\mathrm{H}_{3} \mathrm{O}^{+}\right)$, Zundel $\left(\mathrm{H}_{5} \mathrm{O}_{2}{ }^{+}\right)$and Eigen $\left(\mathrm{H}_{9} \mathrm{O}_{4}{ }^{+}\right)$cations, respectively, often used to model aqueous hydronic species. ${ }^{36}$

As the model catalyst material we employ a nitrogen doped $(14,0)$ carbon nanotube (NCNT), a noble metal-free electrocatalyst that has received notable experimental and computational attention recently. ${ }^{29,33,37-39}$ Specifically, we study hydrogen evolution occurring next to a substitutionally doped, graphitic nitrogen site. This site represents evidently only one of the possible dopant configurations and it has in fact recently been proposed that regarding HER and OER catalysis pyridinic moieties may constitute the most active sites. ${ }^{39}$ Nevertheless, we concentrate on the graphitic nitrogen due to previous research ${ }^{29}$ on this site which we use as a reference, as well as since the main purpose of the present work is to compare the performances of the aforementioned two computational methodologies and the importance of solvent fluctuations, not to elucidate the catalytic activities of the different dopant sites. Although highly interesting, this topic is beyond the scope of the present research and is consequently left for future efforts.

The remainder of this article is organized as follows. In Section 2.1 we briefly review the central theoretical details of the constrained MD and thermodynamic integration formalisms and motivate the chosen reaction coordinates for the Volmer and Heyrovský steps, respectively. Section 2.2 continues with a discussion of the theoretical aspects of a simple chargeextrapolation scheme that we apply to approximate the constant electrode potential behaviour of the as derived energy profiles. Finally, the theoretical methodology is concluded in Section 2.3 with a summary of the employed computational models and parameters. Next, the obtained results are reported and discussed in Section 3, starting first with the static CI-NEB calculations of the Volmer and Heyrovský reactions in Sections 3.1.1 and 3.1.2, respectively. Secondly, the output of the constrained MD simulations and the thermodynamic integration are presented in Section 3.2 and the results are concluded by a critical discussion of the two methods in Section 3.3, highlighting the key differences, advantages and limitations and their implications on electrochemical modelling. The main conclusions are finally summarized briefly in Section 4 .

\section{Theoretical methods}

\subsection{Reaction control by constraints}

As introduced by Sprik and Ciccotti, ${ }^{11}$ the free energy profile related to rare events, such as chemical reactions, can be calculated by introducing a holonomic constraint on a chosen reaction coordinate along which the event under consideration is driven and integrating over the properly unbiased force associated with this constraint. Explicitly, integrating the unbiased, solvent averaged force $\left\langle f_{\mathrm{s}}\left(\xi^{\prime}\right)\right\rangle_{\xi^{\prime}}$ with respect to the specified reaction coordinate $\xi^{\prime}$ yields the relative free energy $\Delta A(\xi)$ at some value $\xi$ on the reaction path according to eqn (3),

$$
\Delta A(\xi)=-\int_{\xi_{0}}^{\xi} \mathrm{d} \xi^{\prime}\left\langle f_{\mathrm{s}}\left(\xi^{\prime}\right)\right\rangle_{\xi^{\prime}},
$$

where $\xi_{0}$ is a chosen reference (initial) state. To obtain the properly reweighted force, one needs to compensate the induced bias in the bare force of constraint $\lambda$, which can be identified as the Lagrange multiplier enforcing the defined constraint. The procedure is defined according to eqn (4),

$$
\left\langle f_{\mathrm{s}}\left(\xi^{\prime}\right)\right\rangle_{\xi^{\prime}}=\frac{\left\langle Z^{-\frac{1}{2}}(\lambda-k T G)\right\rangle_{\xi^{\prime}}}{\left\langle Z^{-\frac{1}{2}}\right\rangle_{\xi^{\prime}}},
$$

where $Z$ and $G$ denote geometric correction terms derived previously ${ }^{11}$ and shown explicitly in the Appendix. Furthermore, $k$ and $T$ correspond to the Boltzmann constant and absolute temperature, respectively. The corrections to the force of constraint are, however, generally negligible as will be demonstrated later in Section 3.2. Thus, eqn (5) holds approximately for a given value $\xi^{\prime}$,

$$
\left\langle f_{\mathrm{s}}\left(\xi^{\prime}\right)\right\rangle_{\xi^{\prime}} \approx\langle\lambda\rangle_{\xi^{\prime}}
$$

The main challenge of constrained MD with thermodynamic integration is that the, often multidimensional, reaction coordinate is generally not known a priori. Thus, a reasonable choice must be based on chemical intuition and tested properly. Briefly, the constrained reaction coordinate should be sufficiently robust so that a required control of the reaction is maintained 
throughout the transition path. If the constraint looses control of the reaction, e.g. after passing the transition state the product desorbs from the surface in an uncontrolled manner and diffuses away, a discontinuity will be observed in the force of constraint. This translates to uncertainties in the derived free energy profile and the reaction mechanism itself. On the other hand, the constraint should be indirect enough to allow for as many degrees of freedom as possible for the reaction to explore the phase space and find a realistic reaction path. For example, defining a simple interatomic distance as a constraint would quench the vibrational motion between the involved atoms.

In the present work, we employ the proton transfer coordinate defined by eqn (6) for the Volmer reaction,

$$
\xi^{\mathrm{V}}(\mathbf{r})=\left|\mathbf{r}_{\mathrm{C}}-\mathbf{r}_{\mathrm{H}}\right|-\left|\mathbf{r}_{\mathrm{O}}-\boldsymbol{r}_{\mathrm{H}}\right|,
$$

where the labeling of atoms is defined in Fig. 1a. This distance difference function is motivated considering the key atoms participating in the reaction, i.e. the adsorption site, the transferring proton and the proton donating oxygen atom. Defining the constraint as a distance difference allows the system to choose whether to decrease the $\mathrm{C}-\mathrm{H}$ distance or increase the $\mathrm{O}-\mathrm{H}$ distance as the reaction is driven forward. While ensuring some flexibility, the constraint still imposes a sufficient degree of control since e.g. the water molecule formed after the reaction is not allowed to diffuse away freely, but is progressively pushed farther from the surface as the $\mathrm{C}-\mathrm{H}$ bond decreases to the equilibrium length.

For the Heyrovský reaction on the other hand we employ the proton transfer coordinate of eqn (7),

$$
\xi^{\mathrm{H}}(\mathbf{r})=\left|\mathbf{r}_{\mathrm{H}}-\mathbf{r}_{\mathrm{H}^{*}}\right|-\left|\mathbf{r}_{\mathrm{C}}-\mathbf{r}_{\mathrm{H}^{*}}\right|-\left|\mathbf{r}_{\mathrm{O}}-\mathbf{r}_{\mathrm{H}}\right| .
$$

where the labeling of atoms is defined in Fig. 1b. This choice was made based on similar balancing of flexibility and control as for the Volmer reaction, but since the number of participating atoms in the Heyrovský reaction is larger a three dimensional distance difference function was employed. Mainly, three distances were required to achieve a controlled approach of $\mathrm{H}^{*}$ and $\mathrm{H}$ followed by steady diffusion of $\mathrm{H}_{2}$ away from both the surface $\mathrm{C}$-site as well as the remaining water molecule.

In practice, however, an apparent issue with simulating proton transfer from an aqueous phase is how to control proton hopping, i.e. the Grotthuss mechanism, so that the proton transfer would truly occur from the solvated complex, not from a water molecule. Two different approaches to mitigate this were tested and the results are detailed in Section 3.2.

\subsection{Constant electrode potential via charge extrapolation}

Considering electron transfer within the context of electrochemical reactions, the potential dependence of reaction energies and barriers is of considerable interest. While no generally accepted, computationally tractable standard procedure for simulating grand canonical, constant electrode potential processes exists, several approximations and extrapolation schemes have been proposed. ${ }^{40-44}$

According to Chan and Nørskov, ${ }^{43,44}$ for simple proton transfer reactions in the absence of adsorbates with substantial molecular dipole moments or considerable solvent reorganization, the electrostatic contribution to the change in energy is purely capacitive and can be separated from the chemical energy change component. Following the proposed simple charge extrapolation formalism, the energy change with respect to a chosen reference state along a reaction path $E_{2}-E_{1}$ at constant electrode potential $U_{1}$ is given by eqn (8),

$$
E_{2}\left(U_{1}\right)-E_{1}\left(U_{1}\right)=E_{2}\left(U_{2}\right)-E_{1}\left(U_{1}\right)-\frac{\left(q_{2}-q_{1}\right)\left(U_{2}-U_{1}\right)}{2} .
$$

a)

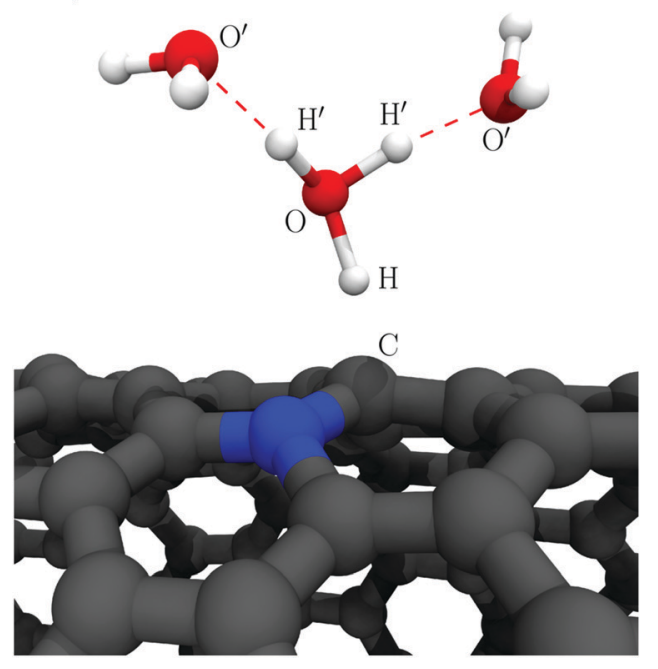

b)

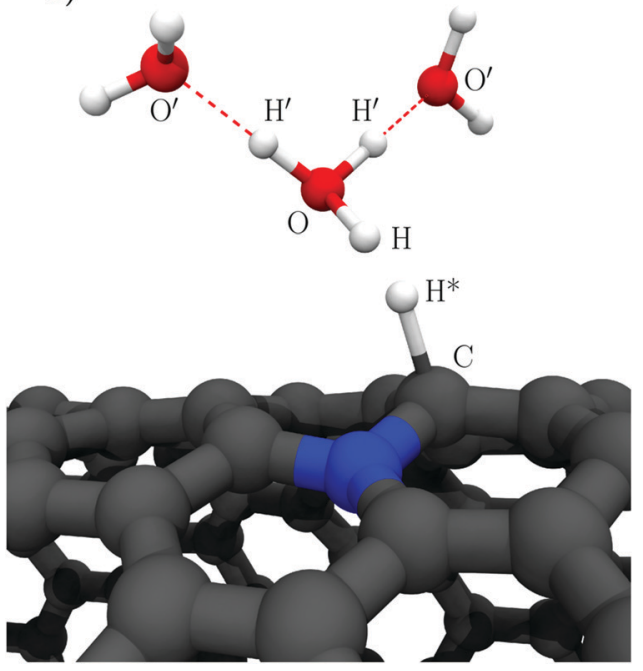

Fig. 1 Illustration of the key atoms and molecular species relevant to the definition of the chosen reaction coordinates of the (a) Volmer and (b) Heyrovský reactions. The example configurations correspond to snapshots from the constrained MD transition state simulations. Water molecules not directly participating in the concerned reactions have been removed for clarity. Gray, blue, red and white spheres correspond to carbon, nitrogen, oxygen and hydrogen atoms, respectively, while hydrogen bonds are indicated by the red dashed lines. 
Here, $q_{i}$ denotes the net charge on the electrode surface in state $i$. Thus, taking for example state 2 as the transition state and state 1 as the initial state, the charge extrapolation scheme yields an estimate of the reaction barrier at the constant electrode potential of the initial state. Analogously, any state along the reaction path can be chosen to obtain estimates of the barrier at different electrode potentials. Conventionally, the absolute electrode potential $U_{\text {abs }}$ is evaluated according to eqn (9), ${ }^{45,46}$

$$
U_{\mathrm{abs}}=\frac{-\mu+e \psi^{\mathrm{s}}}{e}
$$

where $\mu, e$ and $\psi^{\mathrm{s}}$ denote the electrode Fermi level, elementary charge and vacuum (Volta) potential beyond the solvent phase, respectively. To further convert the absolute electrode potential to the experimentally relevant standard hydrogen electrode (SHE) scale, a shift of $-4.44 \mathrm{~V}$ is applied as proposed by Trasatti and recommended by IUPAC. ${ }^{45}$

\subsection{Computational details}

The Volmer-Heyrovský mechanism of HER was studied on a substitutionally nitrogen doped $(14,0)$ single-walled carbon nanotube $\left(\mathrm{C}_{167} \mathrm{~N}\right)$ constructed using ASE. ${ }^{47}$ The model NCNT was solvated using GROMACS ${ }^{48}$ with 201 classically pre-equilibrated SPC water molecules, resulting in a solvent phase with a minimum thickness of $11 \AA$ between periodic copies of the NCNT. In order to model an acidic solution, one excess hydrogen atom was added to the water contact layer close to the nitrogen dopant. This resulted in a $\mathrm{pH}$-value of 0.6 in accordance with $\mathrm{pH}=$ $-\log \left[\mathrm{H}^{+}\right]=-\log \left(x / V_{\mathrm{m}}\right)$, where $x$ is the proton mole fraction $(1 / 201)$ and $V_{\mathrm{m}}$ the molar volume of water $\left(\right.$ ca. $\left.18 \mathrm{~cm}^{3} \mathrm{~mol}^{-1}\right)$. The simulation cell size was $26.00 \times 26.00 \times 12.78 \AA^{3}$. The water density profile between two periodic copies of the NCNT is presented in Fig. 2a (average density $\langle\rho\rangle \approx 0.97 \mathrm{~g} \mathrm{~cm}^{-3}$ ). The high-density liquid (HDL) peak of the water contact layer can be seen to be relatively small, indicative of a hydrophobic character as has been inferred for pristine CNTs. ${ }^{49}$ For calculation of the electrode potential, a vacuum layer of roughly $24 \AA$ was added around solvated NCNT configurations sampled every 100 fs to obtain the vacuum reference level, i.e. the Volta potential. Subsequently, the electrostatic (Hartree) potential of the respective configurations were calculated. An example of the behavior of the average electrostatic potential around the solvated NCNT is shown in Fig. 2b.

All DFT calculations presented in this work were conducted using the CP2K/Quickstep quantum chemistry code. ${ }^{50,51}$ The spin-polarized formulation of the hybrid Gaussian and plane waves (GPW) method $^{52}$ was used with an auxiliary plane wave basis cutoff of $450 \mathrm{Ry}$. Unless otherwise stated, the generalized gradient approximation (GGA) was invoked by applying the exchange-correlation functional of Perdew, Burke and Ernzerhof $(\mathrm{PBE})^{53}$ together with semi-empirical dispersion interaction corrections according to the DFT-D3 scheme of Grimme et al. ${ }^{54,55}$ The damping function of Becke and Johnson was employed ${ }^{56}$ and in addition to the pairwise $C_{6} R^{-6}$ and $C_{8} R^{-8}$ dispersion correction terms the three-body $C_{9} R^{-9}$ term was included. The $2 \mathrm{~s}$ and $2 \mathrm{p}$ electrons of carbon, nitrogen and oxygen and the
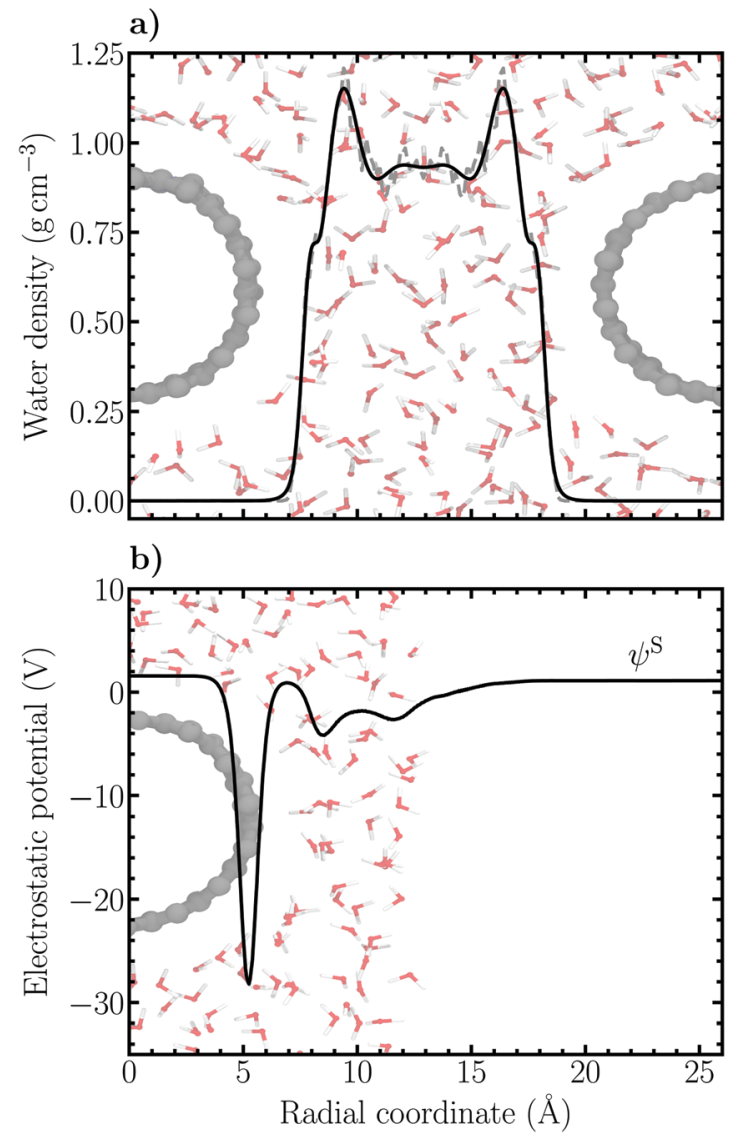

Fig. 2 (a) Water density profile between two periodic images of the NCNT and (b) radial electrostatic (Hartree) potential profile averaged over cylindrical shells centered at the axis of the solvated NCNT. The location of the Volta potential in the vacuum region is indicated. In (a) the density profile has been calculated from the nanotube axis up to the simulation cell boundary $(r=13 \AA)$ and mirrored.

1s electron of hydrogen were considered as valence states. The Kohn-Sham orbitals corresponding to these states were expanded in molecularly optimized double- $\zeta$ plus polarisation quality Gaussian basis sets (MOLOPT-SR-DZVP). ${ }^{57}$ The remaining ionic cores were represented by norm-conserving scalar relativistic Goedecker-Teter-Hutter (GTH) pseudopotentials. $^{58-60}$ The orbital transformation (OT) $\operatorname{method}^{61}$ using direct inversion in the iterative subspace (DIIS) was employed for solving the Kohn-Sham equations. Mixing of the electron density between subsequent iterations was performed using the Broyden method with a $30 \%$ fraction of new density considered each step. A convergence criterion of $2.7 \times 10^{-5} \mathrm{eV}$ for the energy was employed. In performed geometry optimizations the atomic structures were relaxed using the BFGS algorithm until the force on any atom was less than $2.3 \times 10^{-2} \mathrm{eV} \AA^{-1}$.

Static minimum energy paths (MEP) of the Volmer and Heyrovský reactions were optimized using the CI-NEB method. ${ }^{6}$ The respective reaction paths were partitioned into 10 and 12 replicas and optimized until the maximum force reached a value less than $0.1 \mathrm{eV}^{-1}$. The initial and final states of the reactions were prepared and optimized as described previously. ${ }^{29,30,33,35}$ 
To assess the significance of including exact Hartree-Fock exchange (HFX) and the effect of self-interaction error induced electron overdelocalisation on determined activation energies, the CI-NEB images initially converged at the PBE level were reoptimized by single-point calculations using a modified PBE0 ${ }^{62}$ hybrid functional proposed by Guidon et al. ${ }^{63}$ together with the auxiliary density matrix method (ADMM). ${ }^{64}$ Here, the standard HFX energy is replaced by a long-range corrected truncated Coulomb operator (PBE0-TC-LRC). A cutoff radius of $6.0 \AA$ A was employed.

Constrained Born-Oppenheimer MD simulations were performed in the canonical $(N V T)$ ensemble at a temperature of $348.15 \mathrm{~K}$ using a stochastic velocity rescaling thermostat ${ }^{65}$ with a time constant of $100 \mathrm{fs}$. A time step of $0.5 \mathrm{fs}$ was applied. The elevated temperature was chosen to reduce overstructuring of PBE water and to mimic proton quantum nuclear effects that are known to affect the structural and dynamical features of liquid water. ${ }^{6-70}$ We note that the revised PBE (RPBE) ${ }^{71}$ exchange-correlation functional has also recently been demonstrated as a satisfactory alternative in liquid water simulations when applied in conjunction with the semi-empirical DFT-D3 dispersion correction scheme. ${ }^{72,73}$ This functional combination overestimates, however, the oxygen-oxygen distances in the aqueous phase, thereby resulting in a considerably underestimated water density of less than $0.90 \mathrm{~g} \mathrm{~cm}^{-3} \cdot{ }^{74}$ Considering this shortcoming and to allow a better comparison of our results with the ones obtained using the PBE0-D3 hybrid functional as well as the literature, the simulations performed herein were ultimately run using PBE-D3 at the elevated temperature stated above. The SHAKE algorithm ${ }^{75}$ was applied to integrate the Newtonian equations of motion of the systems subject to the defined constraints enforced using the method of Lagrange multipliers as implemented in the CP2K code. Each constrained MD simulation was run for a minimum of $10 \mathrm{ps,} \mathrm{of}$ which the first two picoseconds were considered equilibration and consequently excluded from the analyses. The initial, transition and final states were determined by the constrained MD trajectories which exhibited an average force of constraint of approximately $0.1 \mathrm{eV} \AA^{-1}$ or less.

\section{Results and discussion}

To compare the CI-NEB and constrained MD methods and to consequently investigate the importance of solvent dynamics in reaction path simulations, reference CI-NEB calculations were first performed for the Volmer-Heyrovský mechanism following computational procedures presented elsewhere. ${ }^{29,30,33,35}$ Concisely, the initial state of the Volmer reaction was first fully optimized without atomic constraints. Second, the solvent structure excluding the reacting Zundel cation was frozen to ensure a transition between two minima within the same local environment and to prevent the electrode potential from shifting due to changes in the interfacial molecular dipole. The product state was then optimized with the transferring proton placed on the probed adsorption site and finally the CI-NEB calculation was performed. The same solvent structure was also used for the Heyrovský reaction, i.e. only the reactive species in the initial $\left(\mathrm{H}_{5} \mathrm{O}_{2}{ }^{+}+\mathrm{H}^{*}\right)$ and the final $\left(2 \mathrm{H}_{2} \mathrm{O}+\mathrm{H}_{2}\right)$ states were relaxed. However, in contrast to the previous works wherein a microsolvation (droplet) model was used to model the aqueous phase, we use a fully solvated model for the NCNT-water interface as well as perform further single point calculations at the hybrid PBE0 level of theory to reconverge the electronic structure of the PBE optimized NEB images.

In the following, we first present CI-NEB results for the VolmerHeyrovský mechanism and apply the charge-extrapolation methodology of Chan and Nørskov ${ }^{43,44}$ to approximate the potential dependence of the activation barriers and reaction energies. Subsequently, the constrained MD results and reaction profiles determined via thermodynamic integration are presented and analysed. Finally, a concluding comparison of the performance of the static and dynamic reaction path simulation methods is performed and the implications are critically discussed.

\subsection{CI-NEB}

3.1.1 Volmer reaction. The active adsorption site on which all simulations in this work were performed was chosen based on previously presented results. ${ }^{29}$ Specifically, following substitutional nitrogen doping of the $(14,0)$ CNT, carbon atoms immediately adjacent to the nitrogen dopant are activated such that the hydrogen adsorption energy decreases from $c a .0 .8 \mathrm{eV}$ to $0.0 \mathrm{eV}$. We reiterate that hydrogen adsorption directly to the nitrogen dopant is highly endothermic with a corresponding adsorption energy of approximately $1.2 \mathrm{eV}$ determined using the PBE functional. The energy profiles along the MEP of the Volmer reaction optimized at the PBE level are presented in Fig. 3a. For illustrations of the initial, transition and final state structures, please see the inset images of Fig. $8 \mathrm{a}$.

Employing the herein optimized initial and final states of the Volmer reaction, Fig. 3a shows that the CI-NEB method employing the PBE functional converges to a minimum energy path with an apparent activation barrier and reaction energy of $0.96 \mathrm{eV}$ and $0.74 \mathrm{eV}$, respectively. Although qualitatively similar, the obtained values are somewhat higher than the previous estimates $E^{\ddagger} \approx 0.5 \mathrm{eV}$ and $\Delta E \approx 0.4 \mathrm{eV}$, respectively. ${ }^{29}$ This is the first example of the limitations of the static CI-NEB method for studying reactions occurring at a solid-liquid interface. Indeed, the CI-NEB minimum energy path is highly dependent on the converged initial and final states including the selected solvent configuration. Thus, variations in the initial distances between reacting species and orientation of water molecules will yield significantly differing results for energetic and kinetic parameters. In the present case, the inherent ambiguity in defining the initial and final states results in a final state that is more sterically hindered, or equivalently an initial state that is more stable, than in the previous work. Evidently, due the dynamic nature of the solvent phase, a more reliable estimate of the reaction path and the associated energy profile using CI-NEB would require the optimization of multiple alternative paths. Subsequently, an improved description of the reaction 

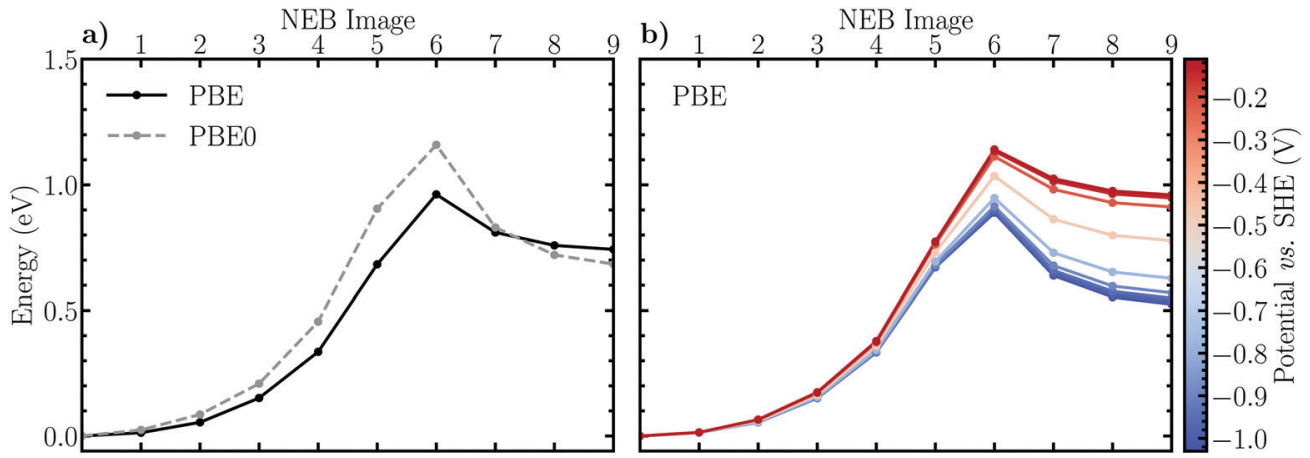

c)

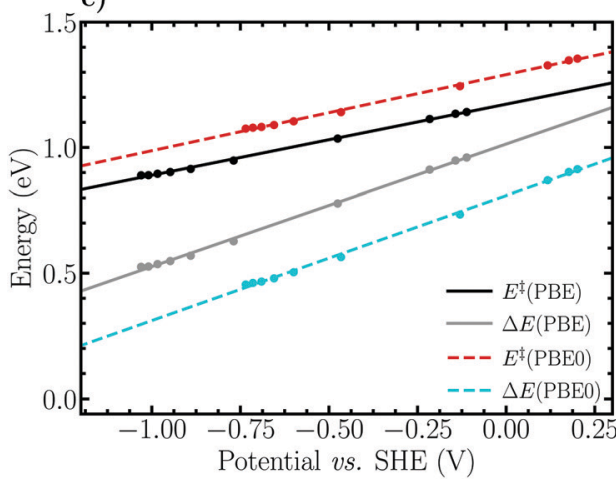

d)

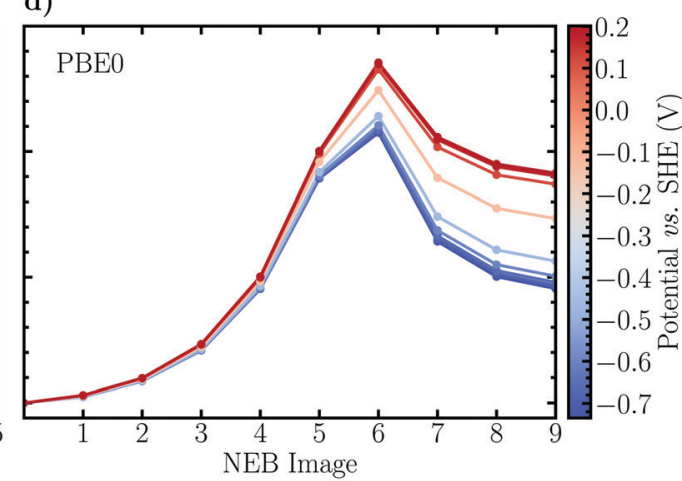

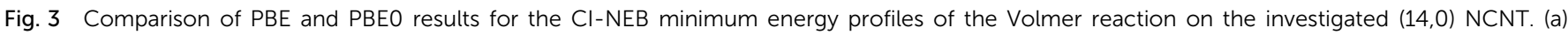

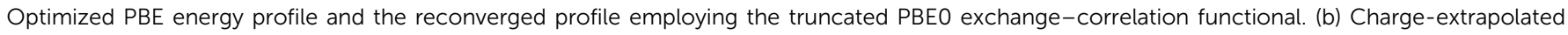

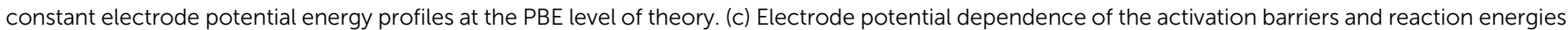

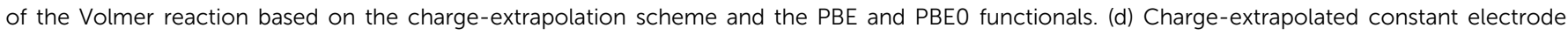
potential energy profiles based on the reoptimized PBEO results.

kinetics and energetics would be obtained via appropriately weighted averages. This qualitative discussion is in line with the reaction rate theory of Chandler et al. ${ }^{76-79}$ who propose that the notion of a single, well-defined minimum energy path should indeed be replaced by the concept of a transition path ensemble. While the formalism of Chandler et al. was not applied in the present work due to its prohibitive computational cost, we note that the constrained MD method of Sprik and Ciccotti is an analogous methodology by which to appropriately sample states along a reaction path. By employing a carefully chosen reaction coordinate, the simulated system is allowed to explore the phase space at each value of the specified constraint. Therefore no single minimum energy path is enforced as in the CI-NEB method, but instead an average constraint force profile and consequently a free energy profile is attained via thermodynamic integration.

Furthermore, Fig. 3a shows that reoptimizing the electronic structures of the converged CI-NEB images using the truncated hybrid PBE0 functional shifts the activation barrier upwards by $0.20 \mathrm{eV}$ and lowers the reaction energy slightly by $c a .0 .06 \mathrm{eV}$. This finding may be interpreted based on the self-interaction error (SIE) inherent in DFT employing (semi-)local functionals. Including exact Hartree-Fock exchange mitigates SIE induced electron overdelocalisation which spuriously stabilizes the reactant and transition states compared to the product state. This conclusion is consistent with earlier studies, ${ }^{80,81}$ in which electron overdelocalisation effects were alleviated by employing a charge-constrained DFT based configuration interaction approach (CDFT-CI). Specifically, in the CDFT-CI study performed on the Volmer reaction on an open-ended CNT, ${ }^{81}$ the barrier height was observed to increase by up to $0.2 \mathrm{eV}$ while the reaction energy in the forward direction was lowered by $0.1-0.2 \mathrm{eV}$.

To obtain a rough estimate of the electrode potential dependence of the minimum energy profile of the Volmer reaction, the charge-extrapolation scheme of Chan and Nørskov ${ }^{43,44}$ was applied. The electrode potential was calculated according to Trasatti $^{45}$ and referenced to the SHE scale while the net atomic charges on the electrode surface were approximated using Hirshfeld population analysis. For further details, please see Fig. S1 and S2 and the associated text in the ESI. $\dagger$ The obtained constant electrode potential energy profiles are presented in Fig. $3 \mathrm{~b}$ and $\mathrm{d}$ for the PBE and PBE0 results, respectively. It appears that the behaviour of the energy profile as a function of the electrode potential is qualitatively reasonable. Indeed, decreasing the electrode potential results in decreased activation barriers and reaction energies due to the fact that the activated complex and product (adsorbed) states are stabilized with respect to the initial state as is expected for electrochemical reduction reactions. To more clearly illustrate how the reaction barriers and energies depend on the electrode potential, these values are plotted separately and shown in Fig. 3c. Concisely, using the PBE functional the activation energies are observed to 
vary between roughly $1.2 \mathrm{eV}$ and $0.9 \mathrm{eV}$ when the potential is decreased from $0.0 \mathrm{~V}$ to $-1.0 \mathrm{~V}$ vs. SHE, while the values for PBE0 are consistently shifted upwards by approximately $0.1 \mathrm{eV}$. We note that a similar degree of potential dependence has been observed previously ${ }^{29}$ for the Volmer reaction using an alternative extrapolation scheme in which multiple CI-NEB energy profiles are calculated for several explicitly charged systems. Using this method, a shift in the activation barrier between $c a$. $0.6 \mathrm{eV}$ and $0.3 \mathrm{eV}$ is observed as the potential decreases from the thermodynamic redox potential of hydrogen, $0.0 \mathrm{~V} v s$. SHE, to $-1.0 \mathrm{~V}$. The constant shift in the barrier heights compared to our work is, however, again present, and is due to differences in the employed frozen solvent and initial state configurations.

Considering the reaction energies obtained using PBE, a decrease from $c a$. $1.0 \mathrm{eV}$ to $0.5 \mathrm{eV}$ within the same electrode potential interval is observed, while the PBE0 results exhibit the same dependence, although shifted downwards by a constant $0.2 \mathrm{eV}$. Evidently, the above discussed difference between the GGA and hybrid descriptions holds qualitatively also following the charge-extrapolation, although an apparent reversal of the magnitude of the energy shifts is seen; the activation barrier increases and the reaction energy decreases by approximately $0.1 \mathrm{eV}$ and $0.2 \mathrm{eV}$, respectively. Finally, the absolute difference between the GGA and hybrid results is observed to be largely constant, i.e. independent of the electrode potential.
3.1.2 Heyrovsky reaction. The static CI-NEB results for the Heyrovský reaction are presented in Fig. 4 in analogy with the previous section. From Fig. $4 \mathrm{a}$ it is observed that the PBE activation barrier and reaction energy are $1.60 \mathrm{eV}$ and $0.83 \mathrm{eV}$, respectively, while the corresponding values following PBE0 reoptimization are $1.83 \mathrm{eV}$ and $0.71 \mathrm{eV}$. We note in bypassing that the final state of the reaction is interpreted as the third to last NEB image based on the fact that this state exhibits the minimum energy on the product side of the reaction. Clearly, the same observations regarding the functional dependence as made for the Volmer reaction can be noted for the Heyrovský reaction as well, i.e. the activation energy increases by $c a .0 .2 \mathrm{eV}$ whereas the reaction energy shifts downward by roughly $0.1 \mathrm{eV}$. However, a careful inspection reveals that the respective energy shifts are up to $60 \mathrm{meV}$ more pronounced for the Heyrovský reaction than for the Volmer reaction. Whether this difference is significant is uncertain considering that the generally quoted DFT error is on the order of $100 \mathrm{meV}$. The observed trend is nevertheless in agreement with the CDFT-CI results presented previously ${ }^{81}$ and suggests that adverse effects due to SIE may be even more pronounced for the Heyrovský reaction.

The potential dependence of the Heyrovský reaction was estimated analogously to the Volmer reaction. Expectedly, the results presented in Fig. $4 \mathrm{~b}$ and d illustrate the same qualitative behaviour, characteristic of reduction reactions. Namely, as the electrode potential decreases (the electrode surface is charged)
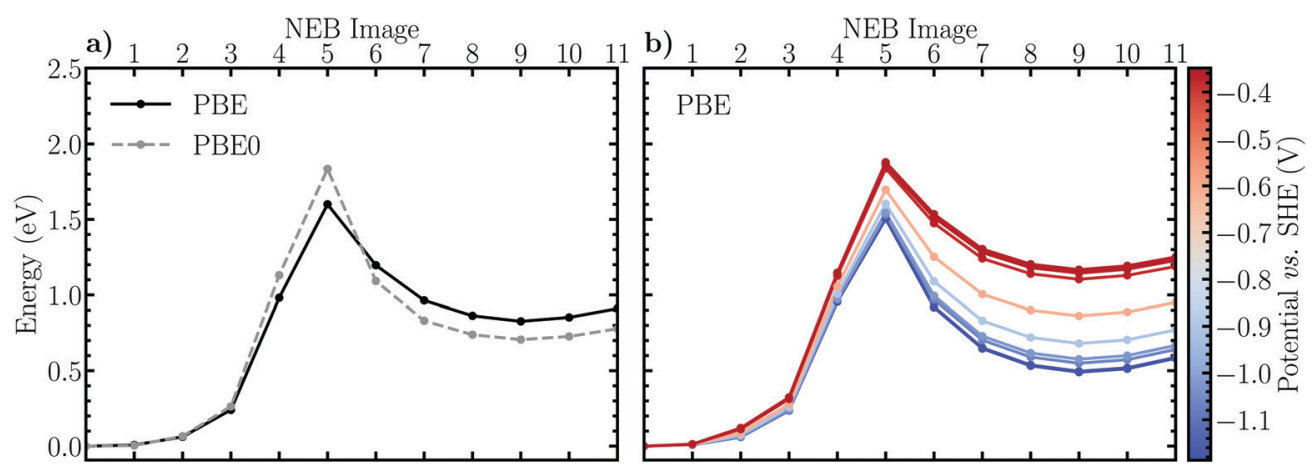

c)

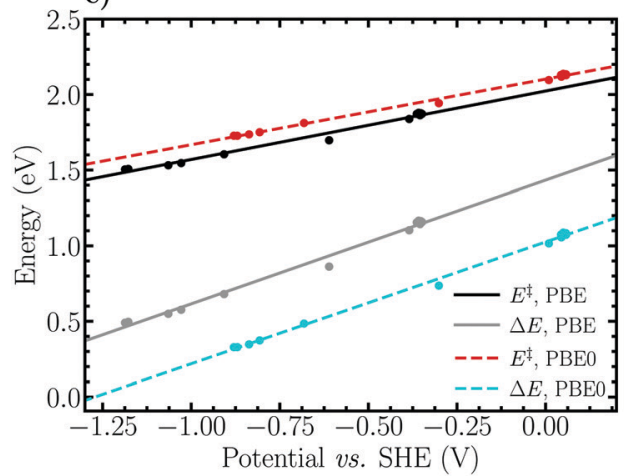

d)

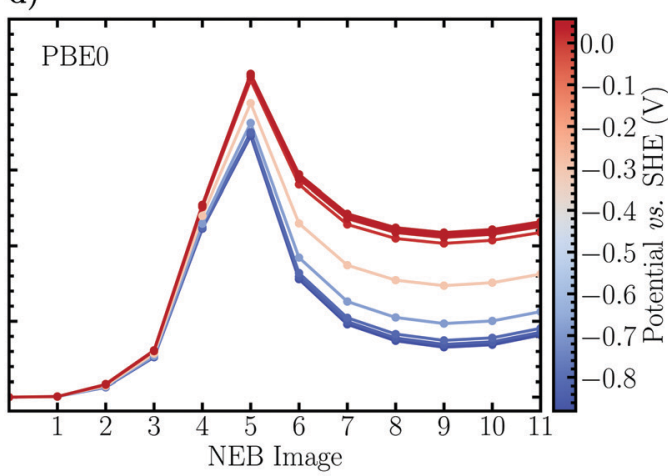

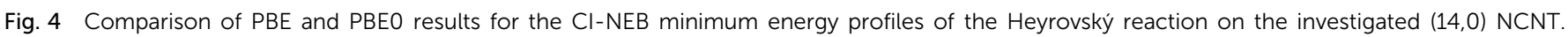

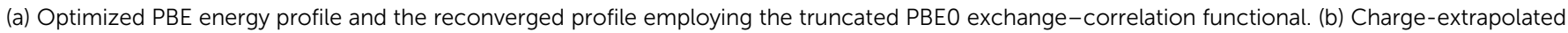

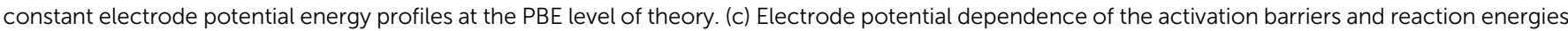

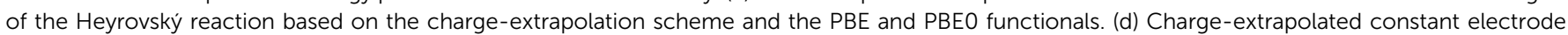
potential energy profiles based on the reoptimized PBEO results. 
the transition and product states are stabilized with respect to the reactant state, thus decreasing the activation barrier heights and reaction energies. The potential dependence of the energetics is observed to be slightly more pronounced than for the Volmer reaction. Concretely, the activation and reaction energies determined using the PBE functional decrease from roughly $2.0 \mathrm{eV}$ to $1.5 \mathrm{eV}$ and $1.4 \mathrm{eV}$ to $0.6 \mathrm{eV}$, respectively, as the electrode potential is decreased from $0.0 \mathrm{~V}$ to $-1.0 \mathrm{~V} v s$. SHE. For the PBE0 energy profiles the corresponding changes are observed between $2.1 \mathrm{eV}$ and $1.6 \mathrm{eV}$ for the activation barrier and between $1.0 \mathrm{eV}$ and $0.2 \mathrm{eV}$ for the reaction energy. The difference between the GGA and hybrid DFT descriptions of the activation barrier is again seen to hold also for the constant electrode potential energy profiles, namely that the PBE0 functional increases the barrier by $c a$. $0.1 \mathrm{eV}$ independent of the electrode potential. The trend for the reaction energies is also analogous, although considerably more pronounced, exhibiting a decrease of approximately $0.4 \mathrm{eV}$ compared to the $0.2 \mathrm{eV}$ observed for the Volmer reaction.

Previous results ${ }^{29}$ obtained using the alternative extrapolation method reveals a somewhat less pronounced potential dependence in which the barrier determined using the PBE functional decreases from $c a .1 .4 \mathrm{eV}$ to $1.1 \mathrm{eV}$ as the electrode potential is varied from $0.0 \mathrm{~V}$ to $-1.0 \mathrm{~V}$. Aside from the constant shift in the barrier height due to differing solvent structures, the potential dependence appears to be nevertheless in a qualitative agreement, providing some support for the computationally lightweight and simple charge-extrapolation scheme of Chan and Nørskov. ${ }^{43,44}$

\subsection{Constrained MD}

Constrained molecular dynamics simulations employing the introduced reaction coordinates (6) and (7) for the Volmer and Heyrovský reactions, respectively, were performed. The force of constraint associated with each value along the reaction coordinate was sampled and averaged over each trajectory and thermodynamic integration over the force profiles was conducted using the cumulative trapezoidal method to obtain the (Helmholtz) free energy profiles of the respective reactions. In order to elucidate the role of proton diffusion, i.e. the Grotthuss mechanism, the Volmer reaction was simulated both in the forward direction of eqn (1) using harmonic $\mathrm{OH}$ restraints to keep the solvated Zundel-like proton complex intact, as well as backward without any additional biasing restraints. For the restrained simulations, a force constant and equilibrium bond length of $19.4 \mathrm{eV} \AA^{-2}$ and $0.99 \AA$ were respectively employed. The applied force constant is roughly $40 \%$ of the force constant of $\mathrm{OH}$ stretching in water. ${ }^{82}$

3.2.1 Volmer reaction. The simulation of proton transfer from an aqueous solution to an electrode surface is complicated by proton hopping via the Grotthuss mechanism. Indeed, performing constrained MD simulations in the forward direction is essentially intractable as the most acidic protons (marked by $\mathrm{H}^{\prime}$ in Fig. 1) may escape from the initial solvated proton complex as the system is propagated. Ultimately, a situation in which proton transfer from a water molecule, not $\left[\left(\mathrm{H}_{2} \mathrm{O}\right)_{n}-\mathrm{H}\right]^{+}$, arises. A simple, although rather unsatisfactory, workaround is to bias the proton complex by imposing restraints on the nearby $\mathrm{OH}^{\prime}$-bonds not involved in the specified reaction coordinate and thus ensure that the proton is transferred to the surface from the hydronic complex. However, another more attractive alternative is to perform the constrained simulations in the backward direction, starting from the adsorbed state. In this case the desorbing proton is continuously well controlled and the Grotthuss mechanism will only occur when the proton has been transferred sufficiently far from the surface, in practice slightly beyond the transition state of the reaction (ESI, $\dagger$ Fig. S5). In other words, there is no need to suppress proton hopping since the diffusion is in any case energetically unfavorable until the reaction has reached a critical extent.

Force and free energy profiles in which both approaches have been employed are shown in Fig. 5 together with error estimates. For consistency, the profiles are shown in the direction of the forward reaction, i.e. large values of the reaction coordinate correspond to trajectories which sample the proton in the aqueous phase while small values correspond to the adsorbed state. The forces of constraint in Fig. 5a are observed to increase gradually as the reaction progresses, reaching a maximum at a constraint value of $0.6 \AA$. Subsequently, the force decreases and passes an inflection point, the transition state, at the value $0.3 \AA$. Following the transition state, the forces are seen to switch sign (direction) and a minimum is reached at $-0.2 \AA$. Finally, the force diminishes, reaching a value less than $0.1 \mathrm{eV} \AA^{-1}$ at $-1.2 \AA$, which is taken as the final state of the Volmer reaction. The shape of both force profiles are observed to be relatively smooth, exhibiting no significant discontinuous jumps between sampled values of the constraint. This suggests that the Volmer reaction remains well controlled over the whole transition path using the chosen reaction coordinate.

It is noteworthy that the constraint force profiles are practically identical for the restrained and unrestrained simulations for trajectories between the transition and product (adsorbed) states. In contrast, restrained trajectories sampling the initial (solvated) states exhibit average constraint forces that are significantly smaller than in the unrestrained simulations. This difference can be understood considering that no Grotthuss hopping may occur when the proton is close to being, or has been, transferred to the surface, and consequently the imposed restraints on the $\mathrm{OH}^{\prime}$-bonds become unimportant. On the reactant side, however, the forces decrease substantially compared to the unrestrained ensemble because the proton complex is artificially stabilized.

Integrating the obtained forces yields the free energy profiles presented in Fig. 5b. We observe that the decreased constraint forces associated with the restrained solvated proton states results in a substantially lower activation free energy, $0.32 \pm$ $0.03 \mathrm{eV}$, and reaction free energy, $-0.52 \pm 0.04 \mathrm{eV}$, compared to the unrestrained free energy profile which exhibits the values $0.73 \pm 0.02 \mathrm{eV}$ and $-0.08 \pm 0.05 \mathrm{eV}$, respectively. The reported error margins correspond to a $95 \%$ confidence interval based on standard errors determined using the method of block averaging and the statistical inefficiency test. Additionally, propagation of 
a)

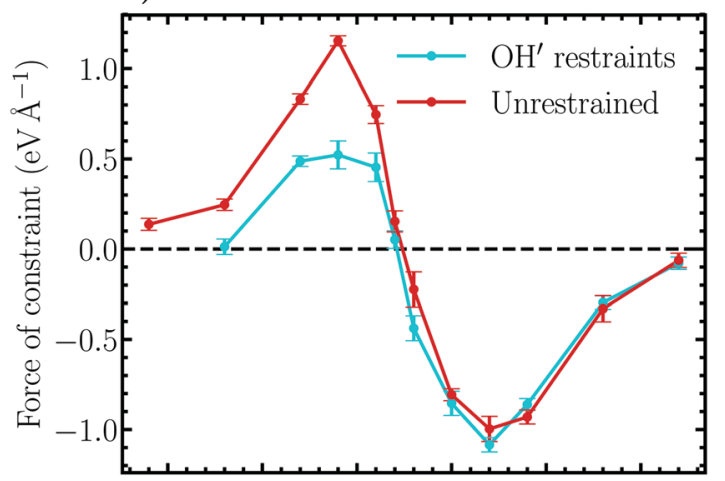

b)

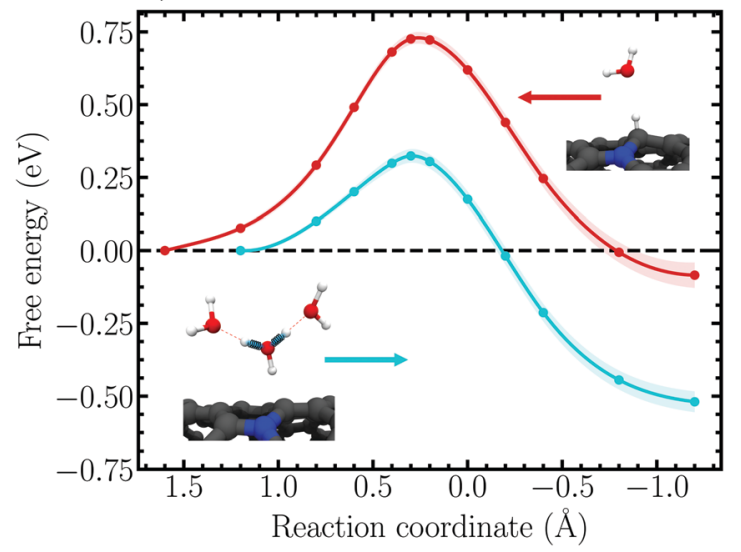

Fig. 5 (a) Constraint force profiles of the Volmer reaction within the restrained and unrestrained ensembles. The error bars indicate $95 \%$ confidence intervals based on standard errors obtained using block averaging. (b) Free energy surfaces along the chosen reaction coordinate (6) of the Volmer reaction within the restrained and unrestrained ensembles. The shaded regions indicate $95 \%$ confidence intervals based on standard errors obtained using the method of block averaging. Propagation of uncertainty within the thermodynamic integration has been accounted for. Note that the legend in (a) refers to both panels. The inset images in (b) delineate the two employed approaches to controlling the Grotthuss mechanism as explained in the main text.

uncertainty within the thermodynamic integration has been properly accounted for. For further details on the calculation of errors, please see Fig. S3 and S4 including the associated text in the ESI. $\dagger$ Interestingly, inspecting the barrier height in the reverse direction reveals that the two parallel simulations yield nearly identical values, roughly $0.8 \mathrm{eV}$. This finding illustrates again the role of the imposed $\mathrm{OH}^{\prime}$ restraints, which play little role on the adsorbed state side of the reaction coordinate, but are decisive for the solvated proton states. Essentially, the initial state is destabilized compared to the transition and final states by $c a .0 .4 \mathrm{eV}$ due to the applied restraints.

To investigate the importance of correcting the bare forces of constraint using eqn (4) to obtain the properly reweighted, solvent averaged force, the free energy surface of the Volmer reaction simulated within the unrestrained ensemble was re-evaluated using the correction terms derived in the Appendix. The result based on the bare force of constraint is replotted together with the unbiased free energy profile in Fig. 6, which

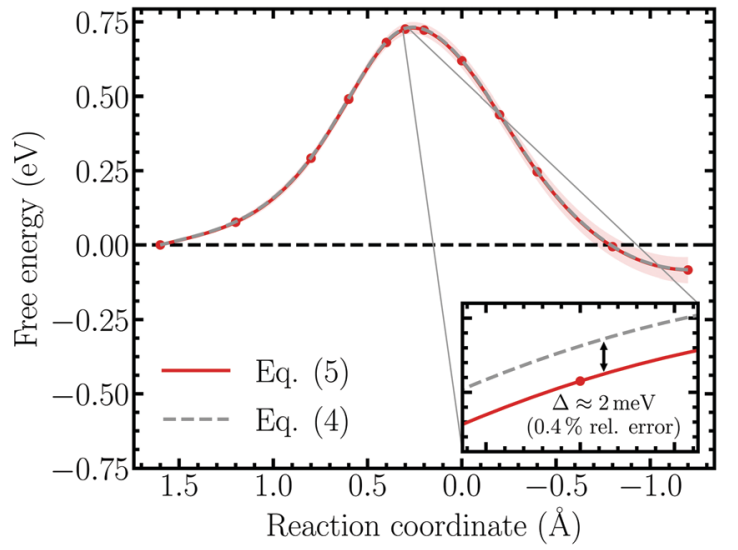

Fig. 6 Comparison of free energy profiles of the Volmer reaction determined from the bare force of constraint, eqn (5), and the unbiased solvent averaged force, eqn (4). The results correspond to simulations performed within the unrestrained ensemble.

indeed demonstrates that the influence of the correction factors is negligible. Specifically, performing thermodynamic integration on the bare force of constraint results in a relative error of roughly $0.4 \%(\sim 2 \mathrm{meV})$, which is well below the accuracy limit of standard DFT.

3.2.2 Heyrovsky reaction. Considering the conclusion that imposed restraints on the solvated proton complex are likely to overbias simulations, the Heyrovský reaction was simulated only in the reverse direction without additional restraints, starting from the $\mathrm{H}_{2}$ product state. The average constraint forces along the reaction path are illustrated in Fig. 7 (forward direction) and follow the qualitatively same trend as the forces in Fig. 5a; the force increases as the reaction progresses forward, passing a maximum at $-1.2 \AA$ followed by the inflection point defining the transition state at roughly $-1.8 \AA$ and leveling out to less than $0.1 \mathrm{eV} \AA^{-1}$ at $-5.0 \AA$ after passing a minimum at the constraint value $-2.4 \AA$. Again, the chosen reaction coordinate is deemed suitable for the purpose of simulating the Heyrovský reaction by considering the continuous, smooth shape of the force profile. Indeed, at no occurrence is the constraint seen to loose control of the reaction which would manifest as a discontinuity in the force of constraint between two subsequent values of the reaction coordinate.

Following thermodynamic integration of the constraint force profile, the free energy surface shown in Fig. $7 \mathrm{~b}$ is obtained. Due to the larger forces of constraint the free energy profile is observed to be steeper than for the Volmer reaction, exhibiting a free energy barrier of $1.56 \pm 0.04 \mathrm{eV}$ and a reaction free energy of $-0.15 \pm 0.07 \mathrm{eV}$. While the reaction free energy of the Heyrovský reaction is nearly identical with the one of the Volmer reaction, the activation free energy is a factor of more than two larger, indicating that the Heyrovský reaction corresponds to the RDS of the hydrogen evolution reaction on the investigated NCNT. This result is in qualitative agreement with the CI-NEB results obtained in this work, as well as in previous studies on similar systems. ${ }^{29,33}$ 
a)

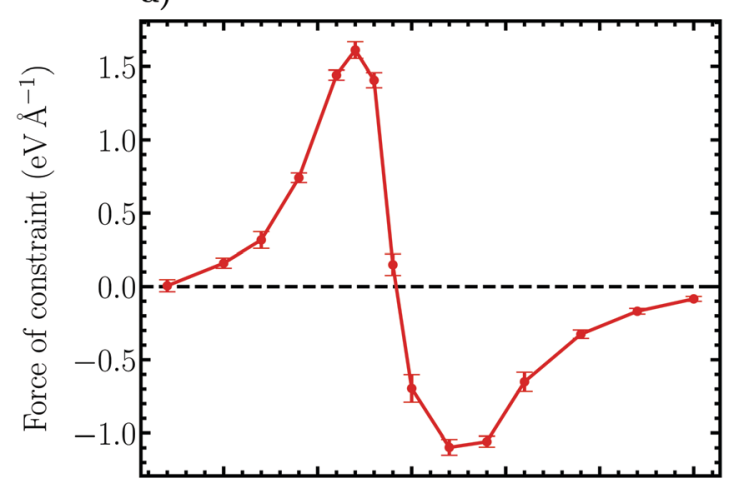

b)

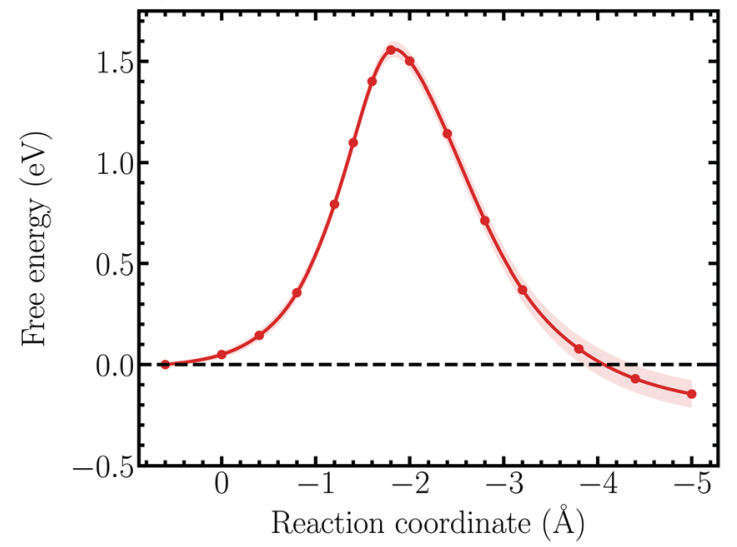

Fig. 7 (a) Constraint force profile of the Heyrovsky reaction within the unrestrained ensemble. The error bars indicate 95\% confidence intervals based on standard errors obtained using block averaging. (b) Free energy surface of the Heyrovský reaction along the defined reaction coordinate (7). The shaded region indicates the $95 \%$ confidence interval based on standard errors obtained using the block averaging method. Uncertainty propagation within the thermodynamic integration has been accounted for.

\subsection{Comparison of CI-NEB and constrained MD results}

3.3.1 Energetics. The activation and reaction (free) energies obtained using CI-NEB and constrained MD with thermodynamic integration are summarized in Table 1 . Note that all tabulated values correspond to canonical (free) energies, i.e. the values have not been corrected for a non-constant electrode potential. While states along a reaction path subject to differing electrode potentials should strictly speak not be compared, the comparison is made between canonical values as the employed charge-extrapolation scheme was found to be inapplicable for the dynamic results. This point will be elaborated in further detail at the end of this section. We thus acknowledge that the listed values may contain uncertainties, although partial cancellation of the variable electrode potential error is also expected. A semiquantitative methodologically consistent comparison is therefore motivated.

Disregarding the dynamic simulations of the Volmer reaction within the restrained ensemble and the hybrid functional calculations, two main observations separates the CI-NEB and constrained MD results. First, the activation barrier heights are lowered by $0.23 \mathrm{eV}$ and $0.04 \mathrm{eV}$ in the case of the Volmer and
Table 1 Activation and reaction (free) energies of the Volmer-Heyrovský mechanism as determined using $\mathrm{Cl}-\mathrm{NEB}$ and constrained MD with thermodynamic integration. The margins of error of the constrained MD results correspond to $95 \%$ confidence intervals based on standard errors obtained using block averaging and accounting for error propagation

\begin{tabular}{llll}
\hline Method & Reaction & $E^{\ddagger} / A^{\ddagger}(\mathrm{eV})$ & $\Delta E / \Delta A(\mathrm{eV})$ \\
\hline CI-NEB, PBE & Volmer & 0.96 & 0.74 \\
& Heyrovský & 1.60 & 0.83 \\
CI-NEB, PBE0 & Volmer & 1.16 & 0.68 \\
& Heyrovský & 1.83 & 0.71 \\
Constr. MD, PBE & Volmer (restr.) & $0.32 \pm 0.03$ & $-0.52 \pm 0.04$ \\
& Volmer & $0.73 \pm 0.02$ & $-0.08 \pm 0.05$ \\
& Heyrovský & $1.56 \pm 0.04$ & $-0.15 \pm 0.07$
\end{tabular}

Heyrovský reactions, respectively. Secondly, the reaction energies decrease substantially, by roughly $0.82 \mathrm{eV}$ and $0.97 \mathrm{eV}$, respectively. Comparing to the PBE0 reoptimized CI-NEB results, the difference between activation energies increases further, while a slight decrease is seen for the reaction energies.

Intuitively, one expects an increase in the activation barrier heights and reaction energies when switching from static calculations to dynamic simulations. Indeed, the static approximations provide an estimate of the minimum energy path and consequently neglect important entropic contributions to the potential energy surface. However, the reason for why this is not observed herein can be understood considering the biased, frozen solvent structure in the CI-NEB calculations. The water structure is optimized in the initial state of the reaction, and because solvent reorganization is suppressed the final state of the reaction will consequently be unphysically strained and therefore high in energy. Now, since the initial and final states of the studied reactions are ultimately connected via the respective transition states, solvent reorganization in the constrained MD simulations will appropriately stabilize the final states and subsequently drag the activation barrier heights downward as well. The fact that the net change in the activation barriers and reaction energies is negative upon inclusion of dynamics indicates thus that stabilizing solvent reorganization dominates over the destabilizing entropic contribution. It is furthermore noteworthy that the herein observed significance of solvent dynamics in the proper description of the Volmer-Heyrovský mechanism has been inferred recently for the Volmer step also using a theoretical PCET formalism accounting for vibrational nonadiabaticity and solvent relaxation dynamics. ${ }^{83}$

Due to the observed considerable influence of solvent reorganization, the Chan-Nørskov charge-extrapolation scheme ${ }^{43,44}$ applied to the CI-NEB calculations to derive energy profiles at constant electrode potentials is deemed inapplicable for the dynamic results. Indeed, since it appears that solvent relaxation dynamics play an important role also in the case of simple proton transfer reactions, the whole underlying notion of describing the electrostatic contribution to the energy change along a reaction path as purely capacitive is placed in a questionable light. For completeness, we have nevertheless calculated the average electrode potentials and surface charges 
for the considered constrained MD trajectories (ESI, $\dagger$ Fig. S6 and S7 and the accompanying text). While the estimated surface charges are found to be comparable with the static results, it appears also that the orientational dynamics of interfacial water molecules induce a heavily fluctuating surface dipole moment that strongly influences the electrode potential ( $0.2 \mathrm{~V}$ standard deviation), resulting in a slow convergence of the average quantity which further complicates a reliable employment of the extrapolation scheme. The magnitude of the electrode potential oscillations agrees with previous simulations..$^{29,84}$ Although the averaged electrode potentials exhibit an increasing trend along the reaction coordinate as expected, the correlation between the surface charge and the electrode potential is weak and the observed slope is significantly lower than in the static calculations. This indicates that solvent fluctuations, reorganization and screening may indeed affect the potential dependence in a decisive manner. Further simulations beyond the scope of this research, such as longer MD runs and application of an alternative constant electrode potential approximation, are however needed for a conclusive say on the matter.

It is nevertheless clear that all obtained activation barriers are irrespective of the applied methodology substantial in magnitude, more than $1.5 \mathrm{eV}$ for the rate-determining Heyrovsky step. This suggests in accordance with previous computational studies predicting Heyrovský reaction barriers of $1.3 \mathrm{eV}$ (singlydoped NCNT), ${ }^{29} 1.2 \mathrm{eV}$ (doubly-doped NCNT) ${ }^{33}$ and $1.2 \mathrm{eV}$ (Fe-encapsulating NCNT) ${ }^{85}$ respectively, that HER catalysis according to the Volmer-Heyrovský mechanism is inefficient on sites adjacent to graphitic nitrogen dopants. Since experimental studies ${ }^{33,39,85}$ nevertheless observe a moderate HER activity ( $c a$. $250-340 \mathrm{mV}$ overpotential at $10 \mathrm{~cm}^{-2}$ ), it is evident that another dopant configuration, such as the pyridinic nitrogen moiety, or a combination thereof is responsible for the activity enhancement. We reiterate, however, that the main purpose of the present work was to contrast the performances of the two aforementioned computational methods and highlight the importance of solvent dynamics, not to elucidate the catalytic activities of various dopant sites.
3.3.2 Structural features. Finally, we compare the structural characteristics of the key reactive species as predicted by the CI-NEB and constrained MD simulations. The obtained reaction paths of the Volmer and Heyrovsky reactions are illustrated using elbow plots in Fig. 8. Additionally, interatomic distances at the reactant, transition and final states according to the applied methodologies are also collected in Table 2 for more precise comparison.

Considering the Volmer reaction (Fig. 8a), a high degree of correspondence between the CI-NEB and constrained MD reaction paths can be observed, although a close inspection reveals a slight "cutting of corners" and sliding of the CI-NEB path. The optimized initial state employed in the CI-NEB calculation exhibits a C-H distance of roughly $2.6 \AA$, a value identical to the constrained MD initial state trajectory. Also at the final state the $\mathrm{C}-\mathrm{H}$ bond lengths are identical, $c a$. $1.1 \AA$, whereas a larger difference of roughly $0.3 \AA$ is seen in the $\mathrm{O}-\mathrm{H}$ distance. This difference is due to the fact that the Volmer reaction had to be driven slightly farther in the dynamic simulation to obtain a sufficiently small average force of constraint. At the transition state, the constrained $\mathrm{MD} \mathrm{C}-\mathrm{H}$ and $\mathrm{O}-\mathrm{H}$ bond lengths are approximately $1.5 \AA$ and $1.2 \AA$, while the corresponding values optimized using CI-NEB are $1.4 \AA$ and $1.3 \AA$, respectively. Evidently, the CI-NEB method predicts a transition state structure in which the adsorbing proton is $c a .0 .1 \AA$ closer to the surface and farther from the donating oxygen than in the constrained MD simulation. This difference illustrates the effect of entropic contributions on the transition state geometry.

Similar conclusions as for the Volmer reaction can be drawn for the Heyrovský reaction path. Here, the final state geometries are practically identical while the initial state $\mathrm{H}-\mathrm{H}^{*}$ bond lengths differ by roughly $0.7 \AA$, the additional distance required to obtain a sufficiently converged reactant state in the dynamic simulation. Importantly, considering the $\mathrm{C}-\mathrm{H}, \mathrm{H}-\mathrm{H}^{*}$ and $\mathrm{O}-\mathrm{H}$ distances, an analogous difference in the static and dynamic transition state geometries of approximately $0.1-0.2 \AA$ is observed. This corresponding behaviour between the Volmer and Heyrovský reactions supports further the above concluded influence of solvent fluctuations, and therefore entropy, on the structural
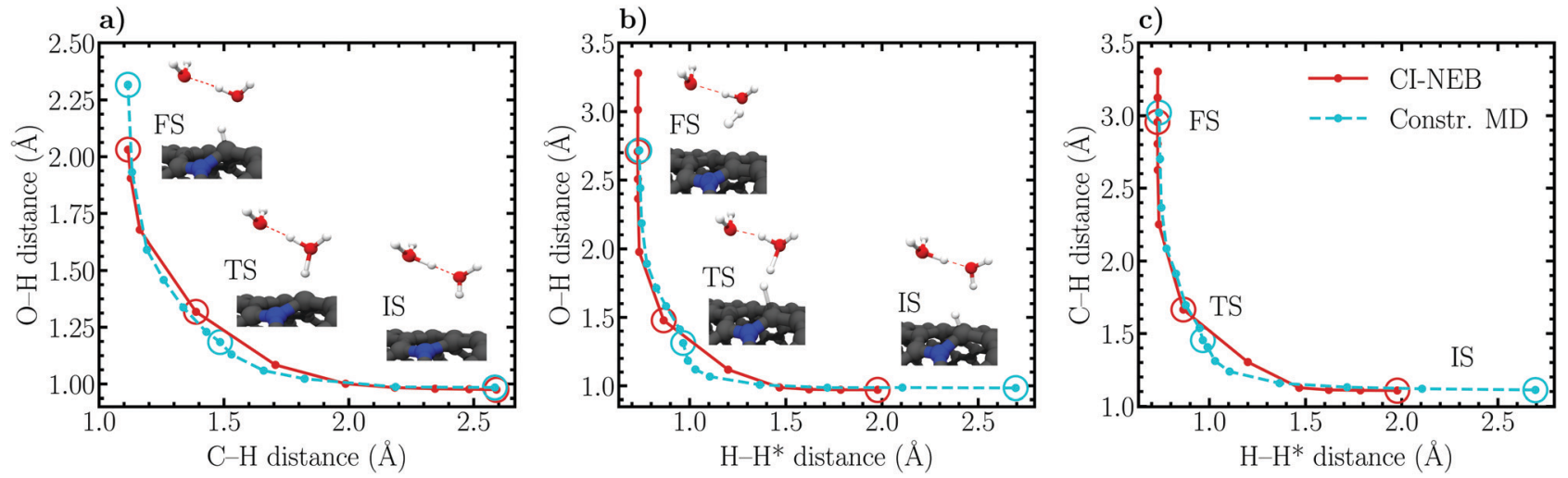

Fig. $8 \mathrm{Cl}$-NEB and constrained MD (unrestrained ensemble) reaction paths of the (a) Volmer and (b and c) Heyrovský reactions illustrated using elbow plots of the interatomic distances composing the employed reaction coordinates. The inset images correspond to the $\mathrm{Cl}$ - $\mathrm{NEB}$ configurations of the respective initial, transition and final states, also highlighted in the data by circles. Note that the legend in (c) refers to all panels. 
Table 2 Structural parameters of the Volmer and Heyrovský reactions as determined using the $\mathrm{Cl}-\mathrm{NEB}$ and constrained MD (unrestrained ensemble) methods. Missing values for the constrained MD initial and final states are due to transport processes (Grotthuss mechanism, water diffusion) that deem the determination of average bond distances meaningless. The atomic labels are as defined in Fig. 1

\begin{tabular}{|c|c|c|c|c|c|c|c|}
\hline \multirow[b]{2}{*}{ Reaction } & \multirow[b]{2}{*}{ Distance $(\AA)$} & \multicolumn{3}{|c|}{ CI-NEB } & \multicolumn{3}{|c|}{ Constr. MD } \\
\hline & & IS & TS & FS & IS & TS & FS \\
\hline \multirow[t]{4}{*}{ Volmer } & $\left|\mathbf{r}_{\mathrm{C}}-\mathbf{r}_{\mathrm{H}}\right|$ & 2.59 & 1.39 & 1.12 & 2.58 & 1.48 & 1.12 \\
\hline & $\left|\mathbf{r}_{\mathrm{O}}-\mathbf{r}_{\mathrm{H}}\right|$ & 0.98 & 1.32 & 2.03 & 0.98 & 1.18 & 2.32 \\
\hline & $\left|\mathbf{r}_{\mathrm{O}^{-}}-\mathbf{r}_{\mathrm{H}^{\prime}}\right|$ & 1.39 & 1.05 & 1.00 & $\mathrm{n} / \mathrm{a}$ & 1.06 & 0.99 \\
\hline & $\left|\mathbf{r}_{\mathrm{O}^{\prime}}-\mathbf{r}_{\mathrm{H}^{\prime}}\right|$ & 1.10 & 1.50 & 1.75 & $\mathrm{n} / \mathrm{a}$ & 1.54 & $\mathrm{n} / \mathrm{a}$ \\
\hline \multirow[t]{5}{*}{ Heyrovský } & $\left|\mathbf{r}_{\mathrm{C}^{-}}-\mathbf{r}_{\mathrm{H}^{*}}\right|$ & 1.11 & 1.67 & 2.96 & 1.11 & 1.45 & 3.02 \\
\hline & $\left|\mathbf{r}_{\mathrm{H}^{-}}-\mathbf{r}_{\mathrm{H}^{*}}\right|$ & 1.98 & 0.87 & 0.73 & 2.69 & 0.97 & 0.74 \\
\hline & $\left|\mathbf{r}_{\mathrm{O}}-\mathbf{r}_{\mathrm{H}}\right|$ & 0.97 & 1.48 & 2.71 & 0.98 & 1.31 & 2.72 \\
\hline & $\left|\mathbf{r}_{\mathrm{O}}-\mathbf{r}_{\mathrm{H}^{\prime}}\right|$ & 1.41 & 1.03 & 0.99 & $\mathrm{n} / \mathrm{a}$ & 1.03 & 0.99 \\
\hline & $\left|\mathbf{r}_{\mathrm{O}^{\prime}}-\mathbf{r}_{\mathrm{H}^{\prime}}\right|$ & 1.08 & 1.58 & 1.84 & $\mathrm{n} / \mathrm{a}$ & 1.61 & $\mathrm{n} / \mathrm{a}$ \\
\hline
\end{tabular}

details of the transition state. Again, while the elbow plots in Fig. $8 \mathrm{~b}$ and $\mathrm{c}$ are relatively similar for the CI-NEB and constrained MD approaches, a slight corner cutting is seen in CI-NEB results. Nevertheless, the ability of the static CI-NEB method to predict a reaction path this close to the dynamically sampled path is rather surprising and exemplifies the good performance of CI-NEB for simulating reactions in environments where entropic effects are insignificant.

Lastly, we briefly discuss the structural dynamics of the reactive hydronic species in accordance with the constrained MD results. While in the CI-NEB calculations the solvated proton complex is conventionally described as a Zundel cation, an Eigen configuration is seen to form in the dynamic initial states. However, in neither the Volmer nor Heyrovský steps does the reaction pass through a transition state involving these species. Instead, an intermediate $\mathrm{H}_{7} \mathrm{O}_{3}{ }^{+}$complex, as illustrated in Fig. 1, is observed where the transferring proton is strongly coordinated towards the NCNT. Specifically, the $\mathrm{H}_{7} \mathrm{O}_{3}{ }^{+}$complex is found to persist at the transition states of the Volmer and Heyrovský reactions as well as in the trajectories immediately following the respective transition states (ESI, $\uparrow$ Fig. S5). However, moving further towards the reactant side of the transition path makes the Grotthuss mechanism energetically feasible and upon transfer of the acidic excess proton farther from the surface the $\mathrm{H}_{7} \mathrm{O}_{3}{ }^{+}$complex decays and an Eigen cation is formed.

\section{Conclusions}

We have performed static climbing image nudged elastic band calculations and constrained molecular dynamics simulations with thermodynamic integration to critically assess the importance of incorporating solvent dynamics when investigating the reaction paths of the Volmer-Heyrovský mechanism of hydrogen evolution on a nitrogen doped $(14,0)$ carbon nanotube. Additionally, the exchange-correlation functional dependence of the CI-NEB energy profiles was examined using the PBE and long-range corrected truncated PBE0 functionals and the electrode potential dependence was elucidated via a simple charge-extrapolation scheme.
In qualitative agreement with previous research, ${ }^{29}$ the PBE activation energy of the Heyrovský reaction was observed to be considerably larger than the barrier of the Volmer reaction, by more than $0.6 \mathrm{eV}$, while the reaction energies were of similar magnitude. Quantitatively, however, the comparison of the reaction energetics is complicated by the employed different static solvent configurations, which in the present work result in a more pronounced stabilization of the initial state. Consequently, the reaction barriers and energies in the forward direction are shifted upwards by roughly $0.3-0.4 \mathrm{eV}$, while the reverse barriers are similar within $0.1 \mathrm{eV}$. Reoptimizing the electronic structures of the PBE CI-NEB images using PBE0 was observed to increase the reaction barriers by $c a .0 .2 \mathrm{eV}$, while reaction energies in the forward direction were found to decrease by approximately $0.1 \mathrm{eV}$. This finding is understood by considering the ability of the hybrid functional to alleviate self-interaction error induced electron overdelocalisation in the initial and transition states and is qualitatively consistent with the results of charge-constrained DFT configuration interaction calculations. $^{80,81}$ Finally, the electrode potential dependence of the Volmer and Heyrovsky energy profiles were found to be qualitatively reasonable and in satisfactory agreement with previous constant potential simulations, taking into account the differences in employed solvent structures resulting in an approximately constant positive shift in the energies of the present work.

The free energy surfaces obtained via thermodynamic integration of the forces of constraint from the constrained MD simulations demonstrated the importance of explicitly sampling the interfacial dynamics when studying hydrogen evolution at a solid-liquid interface. Indeed, the reaction barriers obtained using CI-NEB and the PBE functional were observed to decrease by 0.04-0.23 eV, while even more substantial shifts of up to $-1.0 \mathrm{eV}$ were observed for the reaction energies. The differences between the CI-NEB and thermodynamic integration results were attributed to two opposite effects, namely stabilizing solvent reorganization and destabilizing entropic contributions due to solvent fluctuations. Entropic effects were also found to have an influence on the transition state geometries of the respective reaction steps. Our findings indicating the significance of solvent dynamics when studying interfacial proton-coupled electron transfer corroborate recent theoretical results accounting for vibrational nonadiabaticity and solvent relaxation dynamics. ${ }^{83}$

While a comprehensive computational treatise of electrochemical phenomena is still complicated by the lack of well applicable grand canonical methods, our work exemplifies the importance of incorporating solvent dynamics in the rigorous description of reactions at solid-liquid interfaces and presents an important step towards more accurate electrochemical simulations. The herein applied constrained molecular dynamics simulations employing the proposed reaction coordinates could e.g. be applied to reinvestigate the hydrogen evolution reaction according to the Volmer-Heyrovský mechanism on single-crystal platinum electrodes, a prototypical system where a mismatch between the results of electrochemical experiments and static computational approximations is observed..$^{27,28,86}$ Indeed, this 
defines the topic of our ongoing research with the aim to elucidate how electrochemistry and electrocatalysis should be modelled in order to obtain accurate, experimentally meaningful and, ideally, predictive results.

\section{Conflicts of interest}

There are no conflicts of interest to declare.

\section{Appendix: correction factors to the force of constraint of the Volmer reaction}

The general form of the geometric correction factors $Z$ and $G$ for evaluating the properly unbiased solvent averaged force have been proven by Sprik and Ciccotti, ${ }^{11}$

$$
Z=\sum_{i} \frac{1}{m_{i}}\left(\nabla_{i} \xi\right)^{2},
$$

and

$$
G=\frac{1}{Z^{2}} \sum_{i, j} \frac{1}{m_{i} m_{j}} \nabla_{i} \xi \cdot \nabla_{i}\left(\nabla_{j} \xi\right) \cdot \nabla_{j} \xi
$$

where $m_{i}$ denotes the mass of atom $i$ involved in the specified constraint. Thus, given the proton transfer coordinate of the Volmer reaction in eqn (6), the correction factors are derived as

$$
\begin{aligned}
Z & =\frac{1}{m_{\mathrm{C}}}+\frac{1}{m_{\mathrm{O}}}+\frac{2}{m_{\mathrm{H}}}\left(1-\frac{\left(\mathbf{r}_{\mathrm{C}}-\mathbf{r}_{\mathrm{H}}\right) \cdot\left(\mathbf{r}_{\mathrm{O}}-\mathbf{r}_{\mathrm{H}}\right)}{\left|\mathbf{r}_{\mathrm{C}}-\mathbf{r}_{\mathrm{H}}\right|\left|\mathbf{r}_{\mathrm{O}}-\mathbf{r}_{\mathrm{H}}\right|}\right) \\
& =\frac{1}{m_{\mathrm{C}}}+\frac{1}{m_{\mathrm{O}}}+\frac{4}{m_{\mathrm{H}}} \sin ^{2} \frac{\theta}{2}
\end{aligned}
$$

and consequently

$$
\begin{aligned}
G & =-\frac{\xi^{\mathrm{V}}(\mathbf{r})}{Z^{2} m_{\mathrm{H}}^{2}} \frac{\left|\mathbf{r}_{\mathrm{C}}-\mathbf{r}_{\mathrm{H}}\right|^{2}\left|\mathbf{r}_{\mathrm{O}}-\mathbf{r}_{\mathrm{H}}\right|^{2}-\left[\left(\mathbf{r}_{\mathrm{C}}-\mathbf{r}_{\mathrm{H}}\right) \cdot\left(\mathbf{r}_{\mathrm{O}}-\mathbf{r}_{\mathrm{H}}\right)\right]^{2}}{\left|\mathbf{r}_{\mathrm{C}}-\mathbf{r}_{\mathrm{H}}\right|^{3}\left|\mathbf{r}_{\mathrm{O}}-\mathbf{r}_{\mathrm{H}}\right|^{3}} \\
& =-\frac{\xi^{\mathrm{V}}(\mathbf{r})}{Z^{2} m_{\mathrm{H}}^{2}} \frac{\sin ^{2} \theta}{\left|\mathbf{r}_{\mathrm{C}}-\mathbf{r}_{\mathrm{H}}\right|\left|\mathbf{r}_{\mathrm{O}}-\mathbf{r}_{\mathrm{H}}\right|},
\end{aligned}
$$

where $\theta$ denotes the $\mathrm{C}-\mathrm{H}-\mathrm{O}$ bond angle the vertex of which is defined by the central (transferring) hydrogen atom. The atomic labeling is as defined in Fig. 1a. Correcting the bare force of constraint $\lambda$ by the factors $Z$ and $G$ in accordance with eqn (4) has, however, a relatively insignificant effect on the biased potential energy surface. Indeed, the correction seldom exceeds a few percent as has been discussed by Sprik and Meijer $^{10,12}$ and demonstrated in the present work for the Volmer reaction in Section 3.2.1.

\section{Acknowledgements}

R. K. acknowledges Aalto University School of Chemical Engineering for funding in the form of a doctoral scholarship. Computational resources were provided by the Finnish IT Center for Science (CSC).

\section{References}

1 M. T. M. Koper, J. Electroanal. Chem., 2005, 574, 375-386.

2 M. Nielsen, M. E. Björketun, M. H. Hansen and J. Rossmeisl, Surf. Sci., 2015, 631, 2-7.

3 S. Sakong and A. Groß, ACS Catal., 2016, 6, 5575-5586.

4 R. Jinnouchi, K. Kodama and Y. Morimoto, Curr. Opin. Electrochem., 2018, 8, 103-109.

5 G. Henkelman and H. Jónsson, J. Chem. Phys., 2000, 113, 9978-9985.

6 G. Henkelman, B. P. Uberuaga and H. Jónsson, J. Chem. Phys., 2000, 113, 9901-9904.

7 J. Greeley and M. Mavrikakis, Nat. Mater., 2004, 3, 810.

8 A. Krasheninnikov, K. Nordlund, P. O. Lehtinen, A. S. Foster, A. Ayuela and R. M. Nieminen, Phys. Rev. B: Condens. Matter Mater. Phys., 2004, 69, 073402.

9 B. Qiao, A. Wang, X. Yang, L. F. Allard, Z. Jiang, Y. Cui, J. Liu, J. Li and T. Zhang, Nat. Chem., 2011, 3, 634.

10 M. Sprik, Faraday Discuss., 1998, 110, 437-445.

11 M. Sprik and G. Ciccotti, J. Chem. Phys., 1998, 109, 7737-7744.

12 E. J. Meijer and M. Sprik, J. Am. Chem. Soc., 1998, 120, 6345-6355.

13 M. Sprik, Chem. Phys., 2000, 258, 139-150.

14 N. L. Doltsinis and M. Sprik, Phys. Chem. Chem. Phys., 2003, 5, 2612-2618.

15 P. R. Markwick, N. L. Doltsinis and D. Marx, J. Chem. Phys., 2005, 122, 054112.

16 I. Ivanov, B. Chen, S. Raugei and M. L. Klein, J. Phys. Chem. B, 2006, 110, 6365-6371.

17 P. Carloni, M. Sprik and W. Andreoni, J. Phys. Chem. B, 2000, 104, 823-835.

18 T. Ikeda, M. Hirata and T. Kimura, J. Chem. Phys., 2006, 124, 074503.

19 J. Blumberger and M. Sprik, J. Phys. Chem. B, 2004, 108, 6529-6535.

20 Y. Tateyama, J. Blumberger, T. Ohno and M. Sprik, J. Chem. Phys., 2007, 126, 204506.

21 H.-S. Lee and M. E. Tuckerman, J. Phys. Chem. A, 2009, 113, 2144-2151.

22 J. L. Suter, M. Sprik and E. S. Boek, Geochim. Cosmochim. Acta, 2012, 91, 109-119.

23 J.-C. Chen, B. Reischl, P. Spijker, N. Holmberg, K. Laasonen and A. S. Foster, Phys. Chem. Chem. Phys., 2014, 16, 22545-22554.

24 X. Liu, J. Cheng, M. Sprik, X. Lu and R. Wang, Geochim. Cosmochim. Acta, 2015, 168, 293-301.

25 J. A. Herron, Y. Morikawa and M. Mavrikakis, Proc. Natl. Acad. Sci. U. S. A., 2016, 113, E4937-E4945.

26 C. Zhang, X. Liu, X. Lu, M. He, E. J. Meijer and R. Wang, Geochim. Cosmochim. Acta, 2017, 203, 54-68.

27 E. Skúlason, G. S. Karlberg, J. Rossmeisl, T. Bligaard, J. Greeley, H. Jónsson and J. K. Nørskov, Phys. Chem. Chem. Phys., 2007, 9, 3241-3250.

28 E. Skúlason, V. Tripkovic, M. E. Björketun, S. Gudmundsdóttir, G. Karlberg, J. Rossmeisl, T. Bligaard, H. Jónsson and J. K. Nørskov, J. Phys. Chem. C, 2010, 114, 18182-18197.

29 N. Holmberg and K. Laasonen, J. Phys. Chem. C, 2015, 119, 16166-16178. 
30 N. Holmberg and K. Laasonen, J. Phys. Chem. Lett., 2015, 6, 3956-3960.

31 M. Tavakkoli, N. Holmberg, R. Kronberg, H. Jiang, J. Sainio, E. I. Kauppinen, T. Kallio and K. Laasonen, ACS Catal., 2017, 7, 3121-3130.

32 V. Tripkovic and T. Vegge, J. Phys. Chem. C, 2017, 121, 26785-26793.

33 S. Tuomi, O. J. Pakkanen, M. Borghei, R. Kronberg, J. Sainio, E. I. Kauppinen, A. G. Nasibulin, K. Laasonen and T. Kallio, ChemCatChem, 2018, 10, 3872-3882.

34 L. Partanen, G. Murdachaew and K. Laasonen, J. Phys. Chem. C, 2018, 122, 12892-12899.

35 G. Cilpa-Karhu, O. J. Pakkanen and K. E. Laasonen, J. Phys. Chem. C, 2019, 123, 13569-13577.

36 D. Marx, M. E. Tuckerman, J. Hutter and M. Parrinello, Nature, 1999, 397, 601.

37 Z. Zhang and K. Cho, Phys. Rev. B: Condens. Matter Mater. Phys., 2007, 75, 075420.

38 K. Gong, F. Du, Z. Xia, M. Durstock and L. Dai, Science, 2009, 323, 760-764.

39 F. Davodi, M. Tavakkoli, J. Lahtinen and T. Kallio, J. Catal., 2017, 353, 19-27.

40 J.-S. Filhol and M. Neurock, Angew. Chem., Int. Ed., 2006, 45, 402-406.

41 C. D. Taylor, S. A. Wasileski, J.-S. Filhol and M. Neurock, Phys. Rev. B: Condens. Matter Mater. Phys., 2006, 73, 165402.

42 J. Rossmeisl, E. Skúlason, M. E. Björketun, V. Tripkovic and J. K. Nørskov, Chem. Phys. Lett., 2008, 466, 68-71.

43 K. Chan and J. K. Nørskov, J. Phys. Chem. Lett., 2015, 6, 2663-2668.

44 K. Chan and J. K. Nørskov, J. Phys. Chem. Lett., 2016, 7, 1686-1690.

45 S. Trasatti, Pure Appl. Chem., 1986, 58, 955-966.

46 J. Cheng and M. Sprik, Phys. Chem. Chem. Phys., 2012, 14, 11245-11267.

47 A. H. Larsen, J. J. Mortensen, J. Blomqvist, I. E. Castelli, R. Christensen, M. Dułak, J. Friis, M. N. Groves, B. Hammer and C. Hargus, et al., J. Phys.: Condens. Matter, 2017, 29, 273002.

48 B. Hess, C. Kutzner, D. Van Der Spoel and E. Lindahl, J. Chem. Theory Comput., 2008, 4, 435-447.

49 G. Hummer, J. C. Rasaiah and J. P. Noworyta, Nature, 2001, 414, 188.

50 J. VandeVondele, M. Krack, F. Mohamed, M. Parrinello, T. Chassaing and J. Hutter, Comput. Phys. Commun., 2005, 167, 103-128.

51 J. Hutter, M. Iannuzzi, F. Schiffmann and J. VandeVondele, Wiley Interdiscip. Rev.: Comput. Mol. Sci., 2014, 4, 15-25.

52 G. Lippert, J. Hutter and M. Parrinello, Mol. Phys., 1997, 92, 477-488.

53 J. P. Perdew, K. Burke and M. Ernzerhof, Phys. Rev. Lett., 1996, 77, 3865.

54 S. Grimme, J. Antony, S. Ehrlich and H. Krieg, J. Chem. Phys., 2010, 132, 154104.

55 S. Grimme, S. Ehrlich and L. Goerigk, J. Comput. Chem., 2011, 32, 1456-1465.

56 E. R. Johnson and A. D. Becke, J. Chem. Phys., 2005, 123, 024101.
57 J. VandeVondele and J. Hutter, J. Chem. Phys., 2007, 127, 114105. 58 S. Goedecker, M. Teter and J. Hutter, Phys. Rev. B: Condens. Matter Mater. Phys., 1996, 54, 1703.

59 C. Hartwigsen, S. Gœdecker and J. Hutter, Phys. Rev. B: Condens. Matter Mater. Phys., 1998, 58, 3641.

60 M. Krack, Theor. Chem. Acc., 2005, 114, 145-152.

61 J. VandeVondele and J. Hutter, J. Chem. Phys., 2003, 118, 4365-4369.

62 C. Adamo and V. Barone, J. Chem. Phys., 1999, 110, 6158-6170.

63 M. Guidon, J. Hutter and J. VandeVondele, J. Chem. Theory Comput., 2009, 5, 3010-3021.

64 M. Guidon, J. Hutter and J. VandeVondele, J. Chem. Theory Comput., 2010, 6, 2348-2364.

65 G. Bussi, D. Donadio and M. Parrinello, J. Chem. Phys., 2007, 126, 014101.

66 J. C. Grossman, E. Schwegler, E. W. Draeger, F. Gygi and G. Galli, J. Chem. Phys., 2004, 120, 300-311.

67 J. VandeVondele, F. Mohamed, M. Krack, J. Hutter, M. Sprik and M. Parrinello, J. Chem. Phys., 2005, 122, 014515.

68 H.-S. Lee and M. E. Tuckerman, J. Chem. Phys., 2006, 125, 154507.

69 T. Todorova, A. P. Seitsonen, J. Hutter, I.-F. W. Kuo and C. J. Mundy, J. Phys. Chem. B, 2006, 110, 3685-3691.

70 J. Schmidt, J. VandeVondele, I.-F. W. Kuo, D. Sebastiani, J. I. Siepmann, J. Hutter and C. J. Mundy, J. Phys. Chem. B, 2009, 113, 11959-11964.

71 B. Hammer, L. B. Hansen and J. K. Nørskov, Phys. Rev. B: Condens. Matter Mater. Phys., 1999, 59, 7413.

72 K. Forster-Tonigold and A. Groß, J. Chem. Phys., 2014, 141, 064501.

73 S. Sakong, K. Forster-Tonigold and A. Groß, J. Chem. Phys., 2016, 144, 194701.

74 T. Morawietz, A. Singraber, C. Dellago and J. Behler, Proc. Natl. Acad. Sci. U. S. A., 2016, 113, 8368-8373.

75 J.-P. Ryckaert, G. Ciccotti and H. J. Berendsen, J. Comput. Phys., 1977, 23, 327-341.

76 C. Dellago, P. G. Bolhuis, F. S. Csajka and D. Chandler, J. Chem. Phys., 1998, 108, 1964-1977.

77 C. Dellago, P. G. Bolhuis and D. Chandler, J. Chem. Phys., 1998, 108, 9236-9245.

78 P. G. Bolhuis, C. Dellago and D. Chandler, Faraday Discuss., 1998, 110, 421-436.

79 C. Dellago, P. G. Bolhuis and D. Chandler, J. Chem. Phys., 1999, 110, 6617-6625.

80 Q. Wu, B. Kaduk and T. Van-Voorhis, J. Chem. Phys., 2009, 130, 034109.

81 N. Holmberg and K. Laasonen, J. Chem. Phys., 2018, 149, 104702. 82 Y. Wu, H. L. Tepper and G. A. Voth, J. Chem. Phys., 2006, 124, 024503.

83 Y.-C. Lam, A. V. Soudackov and S. Hammes-Schiffer, J. Phys. Chem. Lett., 2019, 10, 5312-5317.

84 S. Sakong and A. Groß, J. Chem. Phys., 2018, 149, 084705.

85 J. Deng, P. Ren, D. Deng, L. Yu, F. Yang and X. Bao, Energy Environ. Sci., 2014, 7, 1919-1923.

86 N. M. Marković, B. N. Grgur and P. N. Ross, J. Phys. Chem. B, 1997, 101, 5405-5413. 